\title{
LA APLICACIÓN DE POSIBILIDADES TRANSMEDIA E INTERACTIVAS EN LAS PRODUCCIONES SERIADAS DE FICCIÓN DE ANTENA 3: ANÁLI- SIS DE LA ETAPA PLANETA (2003-2010) ${ }^{1}$.
}

\author{
The application of transmedia and interactive possibilities in serial fiction productions \\ of Antena 3: analysis of the Planeta stage (2003-2010).
}

Autora: GARCÍA MIRÓN, Silvia

Doctora - Universidad de Vigo - España-silviamiron@uvigo.es

\section{Resumen}

\begin{abstract}
La emisora privada Antena 3 ha desarrollado en los últimos años un reposicionamiento estratégico basado en los valores de modernidad, innovación tecnológica, participación e interacción (especialmente desde que presenta el proyecto 3.0 en el mes de mayo de 2009), al tiempo que apostaba por la ficción como uno de los macrogéneros claves en su oferta. Desde este punto de partida, nos planteamos con esta investigación destacar las estrategias planteadas por el canal para alargar la vida de sus producciones seriadas de ficción más allá de su emisión en el medio televisivo formulando, por ende, una narración transmedia. Para ello nos detendremos en observar el uso que se hace de la web junto a otro tipo de posibilidades comunicativas como la creación de videojuegos, partiendo de una muestra específica de las series incluidas en el prime time nocturno (principal franja televisiva en términos de audiencia) durante el periodo 2003 (año de inicio de su cuarta y actual etapa empresarial a cargo del Grupo Planeta DeAgostini) y el 2010 (momento en el que se produce el apagón analógico en España). Se plantea, por tanto, como objetivo principal, conocer la adaptación que el medio televisivo está realizando a las nuevas prácticas comunicativas centradas en el consumidor (como sucede con el relato transmedia) así como el empleo de estrategias que nacen vinculadas a aquellas tipologías de NTICs con características interactivas.
\end{abstract}

\section{Palabras clave}

Televisión, ficción, Internet, Antena 3, convergencia multimedia.

\begin{abstract}
The private broadcaster Antena 3 has developed a strategic repositioning based on the values of modernity, technological innovation, participation and interaction in recent years (especially since they decided to introduce the 3.0 project in May 2009), while began to bet on the fiction as the key macrogenre in their offer. From this starting point, we propose with this research to highlight the strategies set by the channel to extend the life of fiction serial productions beyond its broadcast in television media asking, therefore, a transmedia narrative. For this reason, we will observe the use made of the web, as well as other communication possibilities such as creating video games. In order to do this, we use a specific sample of the series included in the nightly prime time (main time strip in terms of audience) for the period 2003 (the year of the beginning of its fourth and current business stage in charge of Grupo Planeta DeAgostini) and 2010 (at which time the ana$\log$ switch off occurs in Spain). Therefore, we establish, as the main objective, to get known about the process of adaptation that the television is making into new communication practices focused on the consumer (as the transmedia narrative) as well as the use of strategies that are related to those types of technologies with interactive features.
\end{abstract}

\section{Key words}

Television, fiction, Internet, Antena 3, multimedia convergente. 


\section{Introducción}

Antena 3 ha desarrollado en sus últimos años una estrategia de reposicionamiento centrado en los conceptos de modernidad, innovación tecnológica, interacción y participación que se orientó esencialmente hacia la convergencia con Internet y la telefonía móvil, especialmente a partir del año 2009 con el proyecto 3.0. No obstante, el modelo programático también se vio afectado creando, en su búsqueda por la satisfacción de las demandas de todo tipo de público, una oferta multigénero obedeciendo de forma clara a su finalidad de emisora de carácter generalista y titularidad privada. Estableciendo una oferta estructurada en torno a dos pilares fundamentales: la información y el entretenimiento (Antena 3 Televisión, 2007), la emisora consigue un buen posicionamiento con el paso de los meses para sus informativos, consolidándose en términos de audiencia y siendo algunos de sus presentadores los más valorados por la audiencia (como ha sido el caso de Matías Prats). Sin embargo, también pone el foco en los concursos, infoshows y ficción (principalmente aquella seriada nacional) como claves de sus contenidos con la finalidad de configurarse como la principal televisión familiar del mercado español. Debemos hacer mención al hecho de que las series de ficción continúan siendo en el contexto del año 2003 una pieza clave en las parrillas y consiguen abrir en estos momentos nuevos huecos en la programación favoreciendo el aumento del consumo televisivo.

Durante la etapa 2003-2010 (que supone su cuarta etapa empresarial bajo la presidencia de José Manuel Lara Bosch y el Grupo Planeta DeAgostini como accionista principal) Antena 3 realizó una fuerte apuesta por las series de ficción nacionales, si bien ya había sido uno de sus pilares desde el momento en que se comprobaron los resultados obtenidos por su producción más paradigmática e identificable, Farmacia de guardia, pero especialmente a partir de la llegada de Antonio Asensio al Consejo de Administración en 1992.

Por otra parte, se advierte cómo el Grupo Antena 3 (actualmente Atresmedia), a través de sus distintas líneas de negocio, está o ha estado presente en los medios de comunicación más relevantes: la televisión (con la TDT), la radio, el cine o Internet. Desde 1993, la Compañía inició un proceso de diversificación, participando en compañías de diversa índole con la finalidad de convertirse en un grupo multimedia y multisoporte audiovisual que le permitiese conseguir distintos objetivos: a) optimizar la estrategia de captación publicitaria para mejorar la cuota de mercado; b) realizar a sus clientes una oferta multisoporte para alcanzar la máxima eficacia de su inversión publicitaria; c) diseñar ofertas comerciales multisoporte para publicidad no convencional; y d) desarrollar y potenciar nuevas líneas de negocio (Antena 3 Televisión, 2003, p. 69). Si bien durante sus distintos ciclos siempre prevalecería la actividad del medio televisivo sobre el resto, desde la etapa Telefónica y especialmente en este último periodo de análisis bajo el liderazgo del Grupo Planeta DeAgostini se resolvió dividir los esfuerzos entre las distintas opciones de actividad económica de las que dispone, más aún teniendo en cuenta que nos encontramos en una época de cambios tecnológicos y de disgregación de soportes y de audiencias por lo que se convierte en urgente descubrir nuevas estrategias orientadas a la captación de inversión publicitaria.

La emisora ha ido incorporando poco a poco las NTICs a sus estrategias de distribución y de comunicación generando narraciones transmedia a partir de sus contenidos de ficción. Debemos considerar por tanto en este punto, la explicación de este concepto a partir de las palabras de Jenkins:

"Cada medio hace lo que sabe hacer mejor, de modo que una historia se puede presentar en una película, puede ser expandida a través de la tele-visión, novelas y cómics y su mundo puede ser explorado y experimentado a través del video-juego. Cada elemento de la franquicia necesita tener entidad suficiente para permitir el consumo autónomo. Es decir, no necesitas haber visto la película para disfrutar del videojuego y viceversa" (Jenkins, 2003).

Lo cierto es que con la llegada de las NTICs se produce un cambio trascendente no sólo en la forma de hacer llegar las creaciones (audiovisuales) sino también en el propio modo de contar las historias. 
"Las estrategias propias de las vanguardias artísticas, ahora son parte de la alteración en los tiempos de la narración, en el juego entre ficción y realidad, en regresiones y digresiones permanentes de las acciones de los personajes, (...). Un contenido puede ser tratado y expuesto en diversos medios con variaciones importantes referentes al mundo de ficción que construyen películas, series de televisión y/o libros" (Pérez y Pérez \& Castellanos Cerda, 2013, p. 68-69).

En este sentido, podemos dejar registro de la relevancia otorgada por Antena 3 a sus contenidos de ficción incluyendo, de ser el caso, nuevas versiones de las historias narradas (Scolari, 2008) en sus series de ficción tras su presentación en la pantalla de televisión o su consumo online (que no deja de ser una misma representación en un consumo diferente en una plataforma también distinta) si bien la acción y los personajes, y el resultado de su combinación, continuarán siendo los ejes sobre los que se diseñe el guión y los que permitan hacer avanzar la historia (Legerén Lago, 2014, pp. 567-582).

\section{Objetivos}

Partiendo de la apuesta del canal por la ficción seriada nacional y su reposicionamiento basado en los conceptos de innovación tecnológica y participación, nos proponemos con esta investigación destacar las estrategias planteadas por Antena 3 para alargar la vida de sus productos de ficción más allá de su emisión en el medio televisivo formulando una narración de carácter transmediático. Para ello nos detendremos en observar el uso que se hace de la web u otro tipo de posibilidades comunicativas como la creación de videojuegos —entendiendo los videojuegos para nuestro estudio desde una perspectiva de narración de historias - con la finalidad de determinar si la puesta en juego de la interacción con el espectador/consumidor y la creación de iniciativas transmedia aplicadas a sus productos más representativos y generadores de valor supondrá una práctica recurren- te en sus estrategias de distribución y comunicación del espacio en cuestión.

Partimos, por tanto, de comprobar por una parte, la creación de posibilidades interactivas ofertadas al telespectador - que podríamos denominar consumidor en este contexto de convergencia multimediaasociadas a las producciones seriadas de ficción nacionales de Antena $3 \mathrm{y}$, por la otra, atender, de ser el caso, al desarrollo de estrategias de narración transmedia.

La investigación se convierte en una suerte de repaso histórico (en concreto, de 7 años, los comprendidos entre 2003 y 2010) sobre la evolución del canal Antena 3 clave para entender el desarrollo que ha experimentado el medio televisivo en España en estos últimos años.

\section{Metodología}

Para llevar a cabo la observación de la estrategia que estas producciones seriadas de ficción nacional siguieron más allá de su emisión televisiva, especialmente (aunque no sólo) en Internet, hemos determinado una metodología de tipo cualitativo por su carácter dinámico (Martínez, 2006) a partir de la utilización de un estudio de caso relevante por sus limitaciones temporales (Coller, 2005) y de desarrollo: las producciones seriadas nacionales de ficción emitidas por Antena 3. El hecho de centrarnos en este canal como representativo de lo que ha ido sucediendo en el contexto televisivo español desde mediados de la década del 2000 obedece no sólo a su importancia histórica y de relevancia social al haber sido la primera cadena privada en empezar las emisiones regulares en España, sino también por haber estructurado a partir de mediados de la década del 2000 su posicionamiento y estrategia de mercado a partir de la convergencia con las nuevas tecnologías y los dispositivos que habían surgido de ellas, buscando de esta forma su ventaja diferencial frente a su principal competencia: La 1 de Televisión Española y Telecinco (principales emisoras pública y privada en España por sus índices de audiencia).

Partiendo de la idea de que "la consecuencia metodológica es que en tanto lo general está contenido 
en lo particular, el análisis de un caso puede dar lugar a conclusiones trascendentes" (Reyes \& Hernández, 2008 , p. 85 ), por lo que podemos determinar que a partir del estudio de este caso específico podrán extrapolarse ideas y características del contexto de Antena 3 a la situación de otras emisoras televisivas.

Por otra parte, hemos establecido la elaboración de una muestra para desarrollar un análisis concreto, para lo cual hemos recurrido a las producciones emitidas en el prime time por ser aquella franja horaria más relevante en términos de audiencia y, por ende, en relación con el presupuesto adjudicado a la compra o producción de los espacios incluidos en este horario y donde se concentran los esfuerzos comunicativos. Una vez seleccionada la franja horaria, hemos abordado una muestra a partir de la selección de la programación correspondiente a cuatro semanas por cada año de análisis, coincidentes con los meses de marzo y septiembre (cambio de temporada de primavera y otoño) y julio y diciembre (representativos de la programación estacional del verano y de Navidad), haciendo un total de 28 semanas de las que extraer la muestra objeto de análisis. A partir de ahí se han extraído los títulos de las producciones seriadas de ficción nacionales que han sido objeto de estudio mediante una técnica de observación directa a partir de una consulta hemerográfica en los principales diarios de tirada nacional y las publicaciones online más representativas del medio televisivo en España, atendiendo a aquellas noticias relacionadas con cada uno de estos espacios y, en concreto, sobre la aplicación de estrategias de interacción o de finalidad transmedia llevadas a cabo por el canal.

Por otra parte, hemos delimitado temporalmente la investigación en el periodo 2003-2010 precisamente por suponer el inicio de una nueva etapa empresarial para el canal bajo la influencia, en este caso, de un grupo editorial; y el final de la era analógica de televisión, ya que a partir del mes de abril del año 2010 únicamente se emite a través de la Televisión Digital Terrestre (TDT).

\section{Evolución empresarial y de posicionamiento de Antena 3 entre 2003 y 2010}

\subsection{Accionistas principales $y$ diversificación empresarial}

Antena 3 integra en esta última etapa lo que se daría a conocer como el Grupo Antena 3 y que se convertiría a partir de marzo de 2013 en Atresmedia tras la fusión con La Sexta. En el año 2003 se convertiría en el primer grupo de comunicación español presente en los negocios de la televisión en abierto y la radio comercial cotizando en los mercados bursátiles españoles. La compañía tenía en este momento entre sus accionistas con multinacionales del sector de los contenidos y de la comunicación como el Grupo Planeta ${ }^{2}$ (del sector editorial), el Grupo DeAgostini (grupo multinacional italiano presente en el ámbito editorial, en los sectores financieros y de seguros y la producción y distribución de contenidos para televisión y cine) o el Grupo RTL (contaba con varias cadenas de televisión y radio en la mayor parte del territorio europeo y la productora de contenidos Freemantle; configurándose como el mayor grupo de comunicación en el continente); a estos, se unía el Banco Santander (primer grupo del sector financiero en España que ocupaba el segundo lugar en Europa como finan- ciador al consumo y también operaba en distintos países iberoamericanos).

José Manuel Lara Bosch es nombrado presidente de Antena 3 y Maurizio Carlotti, Consejero Delegado. Los nuevos accionistas designaron a un nuevo equipo gestor que inmediatamente pondría en marcha un plan estratégico para relanzar a la emisora televisiva apoyándose en cambios en la propia gestión del grupo, necesarios tras la pérdida de 30 millones de euros en el 2002, una crisis de audiencia e incluso podríamos afirmar que de imagen.

La finalidad de la directiva residía en la creación de una emisora rentable, un proyecto que generase valor añadido en el que prevaleciese la adecuación del perfil de la audiencia a las necesidades de los anunciantes por medio de las estructuras de programación (Antena 3 Televisión, 2004). En este sentido, la nueva directiva reorganiza el organigrama en torno a cuatro grandes divisiones: Televisión, Radio, Publicidad y Nuevos Negocios. Buscando esa rentabilidad, el equipo directivo llevó a cabo la reestructuración del grupo y el abandono de ciertas líneas de negocio que no 
ofrecían garantías de crecimiento. Así se siguió un proceso de diversificación mediante la propiedad o participación en las siguientes compañías o filiales: Movierecord, encargada de la gestión de la publicidad que era exhibida en las principales salas de cine (con una cuota de pantalla del 45\% en 2006); Atres Advertising, creada en 2004 con la finalidad de explotar comercialmente los negocios de radio, cine y televisión e incluso introduciéndose en otros mercados como la prensa a través de acuerdos con algunas cabeceras de ámbito regional (Grupo Joly, Diario de Navarra y La Gaceta de Salamanca fueron algunas de ellas); la participación en radio comercial a través de Onda Cero y Europa FM; I3 Televisión, dedicada al desarrollo, instalación y comercialización de aplicaciones y sistemas basados en las TICs; Unipublic, centrada en el patrocinio y marketing deportivo; Antena 3 Eventos; Antena 3 Films, responsable de proyectos audiovisuales; Uniprex TV, filial con la que se gestionaba la televisión local bajo la marca Ver-T; 13 Televisión, dedicada a la comercialización de aplicaciones informáticas y desarrollo de proyectos relacionados con la tecnología y los sistemas de información en medios de comunicación; y Antena 3 Editorial, que editaba y gestionaba los derechos de las sintonías de los programas.

El Grupo apuesta de forma decidida por la comunicación multimedia — si bien ya se habían comenzado a avanzar pasos decididos en esta senda bajo el liderazgo de Telefónica - a través de la confluencia en un mismo mercado de la televisión analógica, la digital, la telefonía (móvil), el cine y la radio, enfocándose hacia el tipo de consumo y formas de entretenimiento seguidas por el telespectador y consumidor de contenidos televisivos actual. Poco a poco, ganaría cada vez más importancia el planteamiento de creación de un modelo de negocio dirigido hacia la comunicación global, hasta que marcaría la estrategia general del grupo otorgando solidez a la marca Antena 3 y principal foco de sus inversiones, señalando en el 2007 que "las nuevas tecnologías, como Internet, y desde luego, las nuevas formas de televisión, ya sean en telefonía móvil o en la inminente TDT, son uno de los campos en los que más esfuerzo, medios y talento estamos invirtiendo" (Antena 3 Televisión, 2007, p. 8), lo que se observaría y demostraría consiguiendo que las emisoras digitales (en aquel momento Neox y
Nova) fuesen, en los primeros años de la TDT, las que mayor audiencia obtuvieron.

Bajo la premisa de no querer hacer frente a los cambios del sector audiovisual sino ser el motor de la nueva industria (Cfr. José Manuel Lara Bosch en Antena 3 Televisión, 2009, p. 3), en el año 2009 se produce el lanzamiento de su nueva concepción empresarial y reposicionamiento en términos de imagen: la estrategia 3.0, respuesta del Grupo a la irrupción de nuevos canales de televisión, a la fragmentación de las audiencias y a los nuevos retos de la comunicación multimedia, y que consistía en conformar una estrategia única y unida mediante una oferta coherente a través de la familia de tres canales del grupo adaptada a las tres pantallas disponibles de visionado (televisión, ordenador y móvil). Asimismo, esta estrategia obedecía a un doble objetivo: atraer la mejor audiencia y satisfacer las necesidades del anunciante, por lo que se crean soluciones innovadoras ${ }^{3}$ para aumentar la eficacia en términos publicitarios.

\subsection{Configurando la convergencia en- tre televisión, Internet y telefonía móvil: Antena 3 Multimedia y lanzamiento del Proyecto 3.0.}

Los momentos iniciales de esta etapa caminarán paralelamente con el desarrollo de una estrategia centrada en ganar mayor cuota de mercado con la finalidad de diferenciarse de la competencia. Las claves se encontraban en ofrecer soluciones globales de comunicación, aglutinando bajo una misma marca un conjunto de soportes que suma a la globalidad de la fuerza de la televisión generalista, la segmentación que aportan Internet, los mensajes a móviles, teletexto, telefonía, bases de datos, etc. y, por otro, en incorporar las nuevas tecnologías a la distribución de información en todas sus modalidades: desde Internet al teléfono móvil (Antena 3 Televisión, 2003, p. 69)

La revolución tecnológica y la aparición de nuevos soportes comunicativos en general y con posibilidad de utilizarse como plataformas de visionado para los contenidos televisivos que ofrecen diferentes fórmulas de participación e interacción, condujeron hacia un cambio significativo en los hábitos y formas de consumo de los telespectadores con repercusión en la actitud que éstos mantienen con respecto a los me- 
dios, desempeñando un papel más activo. Siguiendo esta línea y teniendo en consideración las nuevas fórmulas de acceso a la programación por parte del telespectador, desde el Grupo se plantea la creación de una sociedad, Antena 3 Multimedia, en la que poder integrar el potencial creativo generado en la radio y la televisión hacia las nuevas convenciones de visionado de contenidos audiovisuales (Antena 3 Televisión, 2006, p. 7). Antena 3 Multimedia nace en 2006 con la finalidad de convertirse en el motor de la actividad del Grupo Antena 3 en torno al mundo digital (Antena 3 Televisión, 2006, pp. 26-27) explotando contenidos tanto en soportes comerciales como en otros soportes no convencionales.

En consecuencia, Antena 3 comenzó esta etapa ofreciendo soluciones creativas e interactivas al consumidor a través del teletexto — servicio de información con noticias, resúmenes de prensa, mercadillos, ofertas laborales, el tiempo, la programación o las posibilidades del subtitulado consiguiendo desempeñar una labor social-; la comercialización de licencias de distinta índole (libros, revistas, DVDs, juegos, etc.); el servicio de SMS — a través de ContxtA, que permitía la interactividad entre el espectador y la cadena de televisión y que se había convertido en la marca paraguas bajo la que se habían creado productos y servicios para personalizar el teléfono móvil como un juego sobre Los Simpson o sintonías de las series de ficción de la emisora-; el servicio 303 u $11843^{4}$ de alerta de titulares (noticias, información financiera, sorteos, deportes, etc.) e información, que se amplía durante 2008 al introducirse la apertura de alertas en Orange y el incremento de servicios en Movistar y Vodafone (Antena 3 Televisión, 2008, p. 53); etc. Estas son algunas de las posibilidades complementarias de Antena 3 Multimedia.

Tras haber comenzado a producir varios canales de voz para el portal emoción de Telefónica Móviles en el 2003, junto a desarrollos realizados en WAP, imode y el lanzamiento del primer canal de noticias en MMS (archivos multimedia con texto, voz y fotografía para telefonía móvil, operativo tanto a través de Movistar como de Vodafone) o la realización del canal de actualidad para el proyecto Imagenio de Telefónica de ADSL; nos centraremos, no obstante, en la amplitud de ofertas lanzadas a través de Internet por una parte, y de la telefonía móvil ${ }^{5}$ por la otra, pilares básicos de la estrategia de Antena 3 (y del Grupo) durante la etapa del Grupo Planeta DeAgostini, que conducirá al planteamiento de una visión global que posicione a la emisora a través del proyecto 3.0.

El Grupo disponía su página web como contenedor de todos los programas de la emisora, ofreciendo posibilidad de ampliar la información de las series y programas, con acceso a vídeos y contenidos exclusivos, juegos, posibilidad de chatear con otros fans, etc. La web también se establece como uno de los mayores portales audiovisuales informativos en España a principios de la etapa, ofreciendo las noticias con elementos textuales, gráficos y audiovisuales. En el 2007 lanzaba el nuevo portal de la emisora antena3.com (anteriormente antena3tv.com) reformado y con mayor contenido audiovisual.

A lo largo de 2008 Antena 3 Multimedia se pone en marcha antena3videos.com, lugar de publicación de series y programas de la emisora, consiguiendo incrementar notablemente el número de páginas vistas y de usuarios. La puesta en marcha de antena3videos.com resultó crucial para culminar el proyecto de difusión a través de Internet de series y programas y obtuvo una buena acogida por parte del público. Con esta web se buscaba facilitar al usuario el acceso a todo el material de vídeo disponible de la emisora en la red y que daba opción a diferentes posibilidades en torno a la comercialización de espacios para los anunciantes más allá del preroll (spot previo al visionado del vídeo).

A principios de este mismo año lanza antena3noticias.com, una apuesta por la información con la que se emitía las 24 horas del día información constantemente actualizada a través de Canal A3n24, una plataforma multisoporte que podía ser visionada por IPTV, TDT y por móvil. En el mes de septiembre se estrena la web corporativa grupoantena3.com, reuniendo en una misma web información sobre las distintas empresas del Grupo especialmente operativa para el conjunto de los accionistas.

El Grupo Antena 3 siguió marcando su camino en el proceso de convergencia entre la televisión y los nuevos soportes especialmente a partir del año 2007. 
Continuando la expansión de sus distintas actividades en Internet, en marzo lanza un canal propio en el portal YouTube, convirtiéndose de esta forma en la segunda televisión de Europa en hacerlo (la primera habría sido la británica BBC) y la primera en España. Esta decisión fue seguida a posteriori por el resto de emisoras, si bien Telecinco se resistió e incluso llegó a demandar al portal por incluir fragmentos de sus contenidos (Piña, 2008), lo que influyó en que se convirtiese en una de las principales en cuanto a número de descargas. El uso de Youtube por parte de la cadena tenía una finalidad básicamente de promoción de sus contenidos a nivel internacional. En este año también se crea teleporlared.com, que permitía visionar desde cualquier lugar el canal 24 horas de noticias de Antena 3 Televisión, el Canal Internacional y los dos canales de TDT, Neox y Nova, junto a la transmisión de eventos especiales; en primavera nació otro portal, tucanaltv.com, en formato flash, capaz de alojar un contenido puramente audiovisual; y también se produce el lanzamiento de tuclip.com, un portal que recogía vídeos caseros rodados por los propios espectadores, muchos de los cuales ya habían sido emitidos espacios de la cadena (Antena 3 Televisión, 2007, p. 51).

En el ámbito de la telefonía móvil, el Grupo Antena 3 se configuraría como el primero en España en lanzar canales de tv mobile contando en 2007 con 12 canales que emitían 24 horas diarias implementados en las tres principales operadoras del país (Antena 3 Televisión, 2007, p. 53); mientras que en noviembre abría una nueva vía al firmar un acuerdo con Nokia, para el acceso directo de algunos de los vídeos de la emisora presentes en Internet a través del móvil. Ya en el 2008, destaca la llegada de la señal en directo de Antena 3 a través de Vodafone Live!. Durante 2009 extiende el número de productos de información basado en servicios de mensajería lanzando cinco nuevos canales mobile tv que se sumaban a los doce ya existentes. Por otra parte, se configuró como la primera cadena de televisión en emitir en directo por móvil la señal de televisión así como los primeros en lanzar un canal 24 horas noticias por móvil. En el 2009 también dispuso distintas aplicaciones para dispositivos móviles, desde Iphone (Apple store), Mstore (tienda Telefónica), Tienda Vodafone, Nokia y
Blackberry bajo las marcas antena3.mobi y antena3noticias.mobi.

Poco a poco también va incorporando tácticas con las que facilitar la participación del espectador mediante la utilización de la telefonía móvil para la interacción con los programas mediante sobreimpresiones de su opinión en la pantalla, participando en concursos o sorteos o votando en el curso de una transmisión, lo que se hacía a través de los SMS, SMS Premium y de los números con prefijo 905. Estas posibilidades, junto con el inicio del visionado de sus espacios a través de la web (Cfr. García Mirón, 2012) se entienden como el acercamiento clave a lo que finalmente sería entendido como su nuevo posicionamiento en el mercado a través de la aplicación de valores como innovación, modernidad, interactividad o participación mediante la estrategia 3.0, que se haría efectiva en el año 2009.

La Televisión 3.0 nace de la idea de estar a disposición del espectador independientemente de cómo, cuándo o dónde decida consumir los contenidos de televisión. Ésta era la base del objetivo planteado desde la emisora: adaptar la oferta allá donde estuviese el público. A través de este concepto, Antena 3 se convierte en un operador global de contenidos gestionando estratégicamente tres canales en su introducción (Antena 3, Neox y Nova) a través de tres pantallas (televisión, ordenador y móvil) y se utilizó como base para dar respuesta a los retos que planteaba la comunicación televisiva en este contexto: fragmentación de las audiencia y la pérdida de cobertura publicitaria, mediante la incorporación de la pauta única para la comercialización de los espacios publicitarios (que continúa siendo gestionada a través de Atres Advertising). Estamos hablando del concepto 360 grados, relacionado con el desarrollo de contenidos para diferentes plataformas de una manera sinérgica. Es decir, "que los conceptos y los contenidos que se generan sean susceptibles de distribución y promoción cruzada en todas las plataformas, tradicionales o de punta" (Amoroso, 2009). De esta forma, la emisora rompe con el modelo tradicional de televisión convirtiéndose en un operador global y multisoporte de contenidos al observar que las nuevas fórmulas de consumo televisivo a través de Internet "no sólo no resta audiencia a la televisión, sino que incorpora una 
nueva fuente de ingresos a través de la explotación publicitaria del video online" (Antena 3 Televisión, 2009 , p. 47). Con esta estrategia se incide de forma positiva en la audiencia de las páginas web del Grupo transformándose en un medio en el que generan y se consumen nuevos contenidos y servicios específicos para el público online.

La estrategia 3.0 comprendía un sistema de contenidos cruzados e interconectados para su distribución multicanal y multisoporte, compuesto de un conjunto de herramientas que permitían al espectador navegar por la oferta global de la cadena incluyendo elementos no disponibles hasta la introducción del proyecto: contenidos exclusivos (como el making of de capítulos de sus series, escenas eliminadas, etc.), Watch and Chat (una herramienta mediante la cual los espectadores que se encontrasen visualizando un episodio de una de las series de ficción en antena3.com podían chatear con otros internautas de forma simultánea), entrevistas digitales, redifusión de contenidos, comunidades de fans, el portal www.antena3.mobi (para acceder a los contenidos desde el teléfono), aplicaciones para los dispositivos móviles, el navegador 3.0 o la presentadora 3.0 que anunciaba los contenidos multimedia a disposición del usuario.

A partir de aquí se comienza con una práctica de promociones cruzadas entre Internet y televisión, con numerosas acciones de interactividad en programas y contenidos exclusivos de las series, así como una presencia constante y progresiva en las redes sociales (García Mirón, 2011).

\section{Los contenidos seriados de ficción en el periodo 2003-2010}

En esta cuarta etapa se decide mantener algunas de las producciones del periodo previo, como fue el caso de Un paso adelante (Daniel Écija, Ernesto Pozuelo y Pilar Nadal, 2002-2005) o Policías, en el corazón de la calle (César Rodríguez, Guillermo Fernández Groizard y Salvador Calvo, 2000-2003), recogiéndose en la muestra un total de 18 nuevos títulos entre el año 2003 y el mes de abril de 2010, periodo de análisis.

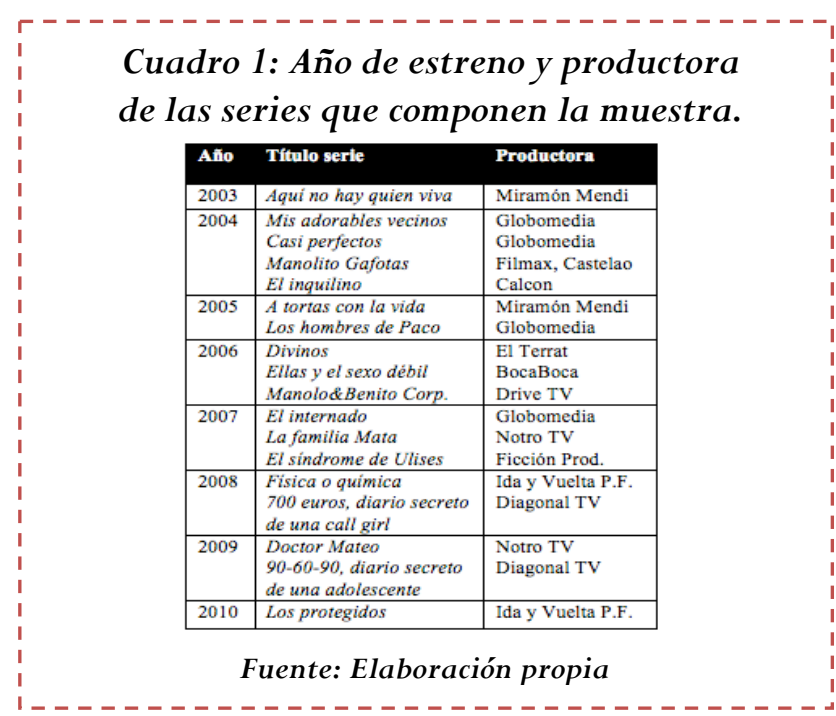

En el año 2003 se presenta a los espectadores una serie que marcaría el sello de la casa durante es-tos años, Aquí no hay quien viva (Alberto Caballero, Laura Caballero, Juan Luis Iborra; 2003-2006), que con un toque realista y un tono humorístico, se formuló como la revelación de la temporada. Aquí no hay quien viva fue una sitcom que narraba las aventuras y desventuras de una comunidad de vecinos. La serie, producida por José Luis Moreno a través de su productora Miramón Mendi, alcanzó una audiencia con la que ni Antena 3 ni el propio reparto de la serie contaban en un principio (Una serie sobre un vecindario, apuesta de Antena 3 para la noche del domingo, 2003, p. 83) consiguiendo convertirse en una de sus producciones seriadas estrella en el horario de prime time por sus cifras de audiencia (Tiempo de seriales. La ficción de producción propia copa la programación televisiva, 2005, p. 7; TVE, en caída libre por octavo mes, 2006, p. 30) logrando una media de 5.354 .000 espectadores, con un $32 \%$ de share en su primera temporada y llegando hasta un $37,1 \%$ en la tercera (Antena 3 inicia la nueva temporada de Aquí no hay quien viva, 2006, p. 10). Tras 5 temporadas pasaría a integrar la programación de Telecinco, manteniendo parte del equipo de guionistas, del equipo técnico e incluso de su reparto.

En 2004 se estrena Mis adorables vecinos (Sandra Gallego, Guillermo Fernández Groizard, César Rodríguez 
Blanco, Gustavo Cotta, Jesús Rodrigo, Pablo Barrera; 2004-2006) y Casi perfectos (Salvador Calvo, Ana Maroto; 2004-2005). Mis adorables vecinos consistía en una comedia familiar, producida por Globomedia, que enfrentaba dos estilos de vida y de ser: una familia de pijos frente a unos horteras e, incluso, lo pretencioso frente a lo llano. De esta forma, toma lo mejor de series históricas como Arriba y abajo y Los nuevos ricos para construir un cuento sobre el conflicto de clases (Antena 3 intenta recuperar el liderazgo del domingo con Mis adorables vecinos, 2004, p. 87). En su estreno se impuso a Siete vidas en Telecinco (también de Globomedia) con 4.900 .000 espectadores que suponía un 30,2\% de cuota de pantalla (El estreno de "Mis adorables vecinos" se impone a "7 vidas", 2004, p. 84), cancelándose tras 4 temporadas. Casi perfectos también estaba producida por Globomedia y presentaba una comedia de corte familiar apta para todos los públicos. Si bien en su primera temporada se habría despedido con un $24,2 \%$ de cuota de pantalla (Emilio Aragón se desmelena en la segunda temporada de Casi perfectos , 2004), en la segundaprogramada contra la serie policíaca El comisario en Telecinco- habría reducido estas cifras y desde Antena 3 se habría optado por no renovarla.

No obstante, en este año también se incorporan varios títulos que no consiguen mantenerse más allá de su primera temporada, como sucedió con Manolito Gafotas (Luis Oliveros, Antonio Cuadri, Antonio Mercero; 2004), basada en la saga de libros de Elvira Lindo que, llegaba a televisión de la mano de Antonio Mercero (creador de Farmacia de guardia); o El inquilino (Paco Arango, Jaime Botella; 2004), que partía de la historia de un extraterrestre que ocupa el cuerpo del escritor Leo Montes (Jorge Sanz), alejándose con esta trama de las series de comedia familiar de corte costumbrista y realista, tendencia en el año 2004 (Antena 3 inicia el rodaje de "El inquilino", su serie marciana, 2004, p. 107).

En 2005 Antena 3 volvía a confiar en la productora de José Luis Moreno para la realización de $A$ tortas con la vida (Alberto Caballero, Laura Caballero, José Luis Moreno, José Antonio Escrivá, Juan Luis Iborra, Rafael de la Cueva; 2005-2006), aunque no obtendría el mismo resultado que el conseguido con Aquí no hay quien viva (A tortas con la vida: Antena 3 vuelve a apostar por el humor de José Luis Moreno, 2005) y finalizaría su vida en televisión tras dos temporadas sin la ejecución de esfuerzos comunicativos reseñables por parte del canal. En 2005 también se incluye otro título que sí conseguirá consagrarse como una de las series de larga duración de la emisora: Los hombres de Paco (Daniel Écija, Álex Pina; 2005-2010). Creada por Globomedia narraba, en tono de humor negro, las vivencias profesionales y personales de un grupo de policías de un conflictivo barrio ficticio de Madrid, personalizándose la historia en el inspector Paco Miranda. Se emitió entre 2005 y 2010 transformándose en uno de los éxitos de audiencia de la emisora con un share comprendido entre el $20,5 \%$ de la primera temporada y un $19 \%$ en su novena y última, con un desgaste provocado por la ausencia de sus protagonistas iniciales y el cambio de la trama en relación con sus inicios (De la gracia en los cataplines, 2007). Con esta serie se produce un paso significativo en relación con la estrategia de mobile $T V$ de la emisora al realizar el preestreno del primer capítulo de la quinta temporada 36 horas antes que en televisión (consiguiendo un 1.300.000 descargas en ese fragmento horario). Con esta táctica también comenzaría a desarrollar nuevas fórmulas de financiación al introducir spots previos al visionado de los vídeos propios de la emisora.

En el año 2006 se apuesta especialmente por el humor para su ficción, pero no acertando con las producciones. Este hecho queda ejemplificado con los casos de Manolo \& Benito Corporeision (Juan Luis Iborra, José Ganga, Jaime Botella; 2006-2007), cuyos resultados fueron desinflándose con cada emisión (Manolo y Benito despiden temporada con su mínimo de audiencia, 2007) y cancelándose tras 2 temporadas; Ellas y el sexo débil (Eva Lesmes, Javier Botella; 2006), que si bien había sido la gran apuesta de Antena 3 para el otoño de 2006, su trama, protagonizada por Ana Obregón e inspirada en determinadas producciones seriadas americanas del estilo de Mujeres desesperadas o Sexo en Nueva York, no consiguió la atención de los telespectadores; o Divinos (Mario Montero; 2006), que a pesar de estar producida por El Terrat y dirigida por el responsable de Siete vidas no lograría cuajar entre los telespectadores y Antena 3 resolvió cancelarla tras emitir únicamente 3 capítulos (Antena 3 retira la serie "Divinos" al tercer episodio por su baja audiencia, 2006, p. 112).

$\mathrm{Al}$ año siguiente se incorporaron nuevas líneas argumentales y nuevos targets. En 2007 y 2008 fue la emisora que más series estrenó y, según GECA, se 
convierte en referencia en ficción. El internado (Daniel Écija, Laura Belloso, Juan Carlos Cuesto; 2007-2010) fue uno de estos casos (y la que mejor rendimiento obtuvo) que apostaba por un perfil de público más juvenil; pero también La familia Mata (Mario Montero, María Pulido, Juan González Gabriel, Luis Santamaría, Ricardo A. Solla; 2007-2009), líder entre el público menor de 24 años; El síndrome de Ulises (Verónica Fernández, Ignacio del Moral, Joan Barbero, Xabi Puerta, Aitor Gabilondo; 2007-2008); Física o Química (Carlos Montero; 2008-2011); o 700 euros, diario secreto de una call girl (David Castillo, Pau Sierio, Jordi C.; 2008).

El internado, producto de Globomedia, tuvo muy buen arranque con más de 4,6 millones de espectadores, alcanzando un share de un 25,3\%, siendo el estreno de ficción nacional más visto desde el 2005 (El internado, el estreno nacional más visto desde 2005, 2007, p. 108). Finalmente la audiencia media se quedaría en el 19,5\% (cfr. datos en el portal de información televisiva online Fórmula TV). La producción — de cuya trama se había advertido ciertos paralelismos con Lost (Jeffrey Lieber, J. J. Abrams, Damon Lindelof; 2004-2010)— consiguió, desde sus inicios, diversos reconocimientos y fue promocionada a través de la comercialización de diverso material de merchandising y licensing. Además de ser editada en DVD, también contó con otro material complementario: una colección de libros editada en Planeta Junior, un juego online y un videojuego de aventura gráfica creado por Virgin Play para Nintendo DS, que llegaría al mercado en la primavera de 2009 coincidiendo con el estreno de la quinta temporada y que se configuraría como la primera serie española en tener un videojuego propio (El internado se convierte en un videojuego para Nintendo DS, 2009).

El internado supuso un enclave estratégico para la emisora, siendo el espacio elegido para el lanzamiento del proyecto 3.0. en mayo de 2009, haciéndose efectivo en el momento que se produjo la emisión en los tres canales del Grupo del primer capítulo de la temporada del año 2009 de esta producción. La novedad radicaba en la emisión transversal de contenidos con productos específicos para cada pantalla. La ficción de Globomedia pudo verse en la televisión analógica con un formato tradicional; en Neox, con un diario de rodaje en la que los actores y el director comenten las escenas; en Internet, donde los usuarios intercambia- ron sus opiniones online que se veían automáticamente en la pantalla de Nova, y, en directo, a través del móvil gracias a la herramienta Watch \& Chat (Antena 3.0 la nueva televisión multimedia, 2009). La iniciativa demostró su éxito, con un $18,8 \%$ de share, 5.000 personas comentando el capítulo por Internet y 300.000 visionados online del capítulo en las horas previas a su estreno televisivo (Antena 3 Televisión, 2009, p. 46).

La familia Mata, por su parte, jugaba con algunos de los elementos propios de la comedia familiar, aunque sus autores imprimían al humor un tono más gamberro. La ficción, producida por Notro TV, creció en sus dos primeras temporadas, con una media de audiencia de 3.037.000 espectadores y un share del $16,9 \%$ convirtiéndose en la segunda opción en su franja de emisión (La familia Mata y una más: Ana Ruiz ficha por la serie de Antena 3, 2008, p. 108). No obstante, con su tercera temporada las audiencias se habían quedado por debajo del $10 \%$ en algunos capítulos, lo que conduce a Antena 3 a decidir su cancelación (Antena 3 finalizará la tercera temporada de La familia Mata con 10 episodios, 2009).

El síndrome de Ulises, de Ficción Producciones, narraba la historia de Ulises (Miguel Ángel Muñoz) un joven médico pijo a quien destinan como coordinador al Centro de Salud Arroyo Pingarrón, en un humilde barrio de Madrid. Sería cancelada tras 3 temporadas con un evidente descenso de audiencia: la media de la primera temporada había sido de un 16,2\% de cuota, con lo que había logrado imponerse a las demás ofertas (Fernando Tejero salta a Antena 3 y ficha por El síndrome de Ulises, 2008, p. 105), sin embargo la última se había quedado en un 12,2\%.

Física o química se estrenaba en febrero de 2008 y lograría mantenerse en la programación tras 7 temporadas. La acción era la propia de una serie coral de adolescentes y su convivencia en un instituto. Tuvo una audiencia media de 2.679.000 telespectadores en todas sus temporadas, un 15,5\%, partiendo de unos buenos datos iniciales y descendiendo su número de telespectadores de forma progresiva hasta situarse en su última temporada en un share medio de un 10,7\%. Esta serie fue objeto para idear una nueva estrategia en relación con la mobile $T V$, mediante la realización del preestreno en Internet y también en dispositivos de telefonía móvil de Física o Química (Carlos Monte- 
ro; 2008-2011), generando casi 2.200.000 descargas y 20.000 accesos en Vodafone Live!

Por último en 2008 nos encontramos el caso de 700 euros, diario secreto de una call girl, una serie de final cerrado que trata el tema de la prostitución de lujo. Programada en el prime time del martes durante el periodo estival del 2008, obtuvo una audiencia media de 2.353 .000 espectadores que supuso un $17,2 \%$ de cuota de pantalla.

En 2009 sobresale Doctor Mateo (César Rodríguez Blanco, Ángeles Reiné, Jaime Botella, Enrich Folch, Manuel Tera; 2009-2011) por su liderazgo en la ficción nacional de las noches de los domingos. Se basaba en la serie Doc Martin emitida originariamente en Reino Unido (Antena 3 estrena en enero la serie Doctor Mateo, 2008). La trama pone el foco en el doctor Mateo Sancristóbal, un cirujano con escasas habilidades sociales que regresa de EEUU para ejercer la medicina en un pequeño pueblo de Asturias y comenzará allí una nueva vida. La serie captó el interés de la audiencia desde un principio, logrando diversos premios y reconocimientos (TP de Oro, Ondas, Mejor Ficción de Comedia en el FesTVal, etc.), y se mantuvo en emisión un total de 5 temporadas. Su media de audiencia, en el cómputo total de sus episodios, fue de 2.674.000 telespectadores, que supone un $14,6 \%$ de cuota de pantalla (El último capítulo de Doctor Mateo este domingo, 2011).

Otro de los estrenos en 2009, si bien con un seguimiento menor, fue 90-60-90. Diario secreto de una adolescente (Diego Lesmes, Miguel Conde, Joan Noguera; 2009) producida por Diagonal TV (al igual que 700 euros, diario secreto de una call girl), que ponía el foco de atención sobre los distintos aspectos del mundo de la moda desde la perspectiva de una adolescente.

En 2010, antes del cierre de la muestra, se observa la inclusión en la parrilla de otra serie que marcaría el carácter familiar de la emisora: Los protegidos (Ignacio Mercero, Ruth García, Darío Madrona, David Lorenzo, David Oliva, Luis Gamboa, Alexandra Olaiz;
2010-2012), que se mantuvo durante 3 temporadas. Producida por Ida y Vuelta P.F., su acción se centra en Mario y Jimena cuyos hijos poseen ciertos poderes especiales y acabarán formando una falsa familia acogiendo a otros chicos y chicas con poderes. La serie, con su combinación de drama y fantasía, logró convertirse en una de las revelaciones de la temporada 2009-2010, finalizando con una media de un 15,1\% de share, si bien experimentando una evolución negativa desde su primera temporada, con 3.361.000 telespectadores y un $18,1 \%$ de cuota de pantalla (Vuelve la serie Los protegidos, 2012, p. 102). Había sorprendido con su capítulo inicial al ser rodado en 3D - la técnica estereoscópica favorecía aquellas secuencias en las que se mostraban los poderes de los personajes - especialmente para ser preestrenado en salas cinematográficas, conjuntamente con centros escolares y hospitales.

La serie había tenido una gran acogida en su versión web donde había ofrecido a lo largo de toda la temporada contenidos exclusivos (Antena 3 emite esta noche el final de Los protegidos, 2012) y disponía de un juego online. Así, coincidiendo con la emisión del capítulo final de la serie, los seguidores de Los protegidos tuvieron la oportunidad de acceder, desde antena3.com a un montaje exclusivo, en vídeo, en el que resumían los acontecimientos más destacados de las tres temporadas de la serie. También se incluía una selección de los momentos más románticos y se ofrecían imágenes inéditas del making of del último capítulo y un final alternativo escrito por los guionistas, el diario de rodaje, una sección denominada "Cómo hemos cambiado desde que empezó la serie hasta ahora" y otra sección con la relación de todos los poderes de los personajes acompañada de una encuesta en la que se elegía el favorito de la audiencia.

Como una de las acciones a través de la sinergia entre la emisora y una red social, se produjo el preestreno en primicia de un avance en Tuenti (red social española líder en el target entre los 14 y los 18 años) (Antena 3 Televisión, 2009, p. 48).

\section{Discusión}

En este texto hemos descrito y ejemplificado cómo las tecnologías digitales han sido un elemento esencial para las emisoras televisivas a la hora no sólo de distribuir sus contenidos sino también de ofrecer fórmulas diferentes de consumo de sus propios contenidos al espectador creando posibilidades interactivas o ofertando narrativas transmedia de sus series de ficción en formatos clásicos como libros o en forma de videojuegos online (alojados en sites propios de la cadena o creados específicamente para este fin) o para 
videoconsolas (como es el caso de la Play Station), así como apps para telefonía móvil.

Desde el repaso realizado advertimos cómo el Grupo Antena 3 decide mantener su posición en el campo de los contenidos multiplataforma, al conseguir aportar una imagen actual e innovadora, al tiempo que trataba de atender a la demanda de una audiencia cada vez más exigente y se ofrecían nuevas ventanas y opciones comunicativas interesantes para el conjunto de los anunciantes (Antena 3 Televisión, 2008, p. 52). La innovación tecnológica aquí aplicada se compatibilizó con la ejecución de tácticas con las que favorecer o facilitar la participación del espectador, con lo que consigue consolidarse como una emisora interactiva. En este sentido, se opta por vincular los contenidos programáticos de Antena 3 con el perfil multimedia, asociando los estrenos a la red o a la telefonía móvil como una fórmula de incentivar a los telespectadores hacia la utilización de las nuevas plataformas de consumo audiovisual propias al tiempo que se amplía la oferta de opciones de interacción ${ }^{6}$. La mobile TV se configuró como uno de los sectores con mayor evolución durante estas décadas y así, Antena 3, trató de adoptar una posición de abanderada ante el desarrollo tecnológico, ya fuese a través de vídeo bajo demanda como señales en directo o así como la posibilidad de visionado en bucle o loops. En este sentido, debemos hacer constar que en mayo de 2007 abría sus producciones seriadas a la red, emitiendo en abierto los capítulos completos de títulos como El internado, Los hombres de Paco, La familia Mata o El síndrome de Ulises, consiguiendo una buena respuesta tanto de la audiencia como por parte de los anunciantes. Desde este momento, la posibilidad de consumo online en directo o a la carta se convertiría en una opción permanente para el espectador.
Por otra parte, en la presente revisión por las estrategias de posicionamiento y distribución de Antena 3 se ha advertido como Internet y la telefonía móvil han sido las dos bazas por las cuales ha apostado la cadena en los últimos años y así ha dejado constancia con los pasos que ha seguido en este sentido, siendo la primera cadena española que creaba su propio canal en el portal Youtube, realizando preestrenos a través de la web desde el año 2008 con algunas de sus series de mayor éxito o incluso siendo pionera en emitir en directo su programación a través de móvil $3 \mathrm{G}$. Uniendo ambas fórmulas comunicativas junto con la ya tradicional emisión por el televisor convencional, Antena 3 decidió lanzar al mercado su nueva apuesta estratégica: el proyecto multimedia 3.0.

También se ha podido determinar cómo desde la División Multimedia de la Compañía se incide en la estrategia multisoporte: páginas web, presencia en los portales audiovisuales (Youtube), creación de perfiles en redes sociales (Facebook, Tuenti, Haboo, Windows Live Messenger, etc.), aplicaciones para telefonía móvil, videojuegos online, diseños desarrollados para estar presente en la oferta de las videoconsolas (Play Station o Nintendo) o en los televisores de nueva generación. Es por ello que el otro gran eje de Antena 3 (y por asociación, del Grupo) en Internet ha sido una creciente presencia en redes sociales (Garcia Mirón, 2011) acompañada de la integración de herramientas de comunidad y socialización en sus webs. La plataforma social en la que mejor se integró Antena 3 desde sus inicios ha sido Facebook junto con Windows Live para la gestión de la interactividad y posibilidad de chat en la página web. Sin embargo, las posibilidades de interacción permitidas por el canal han ido más allá de la presencia en redes sociales, potenciándose los blogs, los encuentros digitales y las retransmisiones online.

\section{Conclusiones}

En el análisis de la interacción y las posibilidades transmedia que lleva a cabo Antena 3 Televisión con sus producciones seriadas de ficción de procedencia nacional, nos encontramos que varios casos en los que sí se crean fórmulas en este sentido. Así, podemos destacar la estrategia utilizada con El internado, serie elegida para realizar el lanzamiento del proyecto Antena 3.0, el desarrollo de videojuegos online de este mismo título o de Los protegidos.

Por otra parte, también resulta significativo hacer constar el hecho de que las series que mejores resulta- 
dos han dado al canal, al tiempo que han sido aquellas que han trascendido a la audiencia más allá de su emisión en televisión pudiendo interactuar o ser partícipes de distintas opciones, han sido aquellos títulos producidos por Globomedia (Los hombres de Paco, El internado) e Ida y Vuelta P.F (Física o Química, Los protegidos). En consecuencia, también puede determinarse que el empleo o desarrollo de este tipo de tácticas se desarrolla con carácter general para contenidos dirigidos a un público más joven susceptible de ser target para emplear e interactuar con las aplicaciones desarrolladas.

Por último, queremos dejar constancia de que este no ha sido un camino con inicio y fin, sino que desde el año 2010 han proliferado nuevos casos y nuevas posibilidades interactivas a partir de las producciones seriadas de ficción del canal, como ha sido el caso de Hispania, la leyenda (Ramón Campos; 2010-2012) que ofrecía una narración complementaria a través de un videojuego online o El barco (Álex Pina, Iván Escobar; 2011-2013) cuya trama se completaba no sólo con un videojuego al igual que el ejemplo anterior sino que se posibilitaba la interacción de los seguidores de la serie mediante las redes sociales a través de lo que han venido en denominarse como twittersodios, una fórmula para crear expectación en torno al producto y que consistían en breves historias que completaban o prolongaban la acción más allá de la emisión en televisión y se hacía a través de Twitter. De esta forma podemos determinar que la permanencia y continuidad de esta tipología de estrategias de distribución y desarrollo de las narraciones de la ficción que es emitida inicialmente (si bien no de forma obligatoria en el actual contexto, tal y como hemos comprobado) a través de la pantalla televisiva.

\section{Notas}

1. La investigación forma parte del trabajo desarrollado para la tesis doctoral de la autora que lleva por título "Antena 3, nacimiento y evolución (1990-2010). Contenidos, estilo y estrategias de programación en prime time de la primera emisora de televisión privada española" que fue defendida el 3 de abril de 2014.

2. El Grupo Planeta, primer grupo editorial del mundo en lengua española por volumen de ventas en el año 2003 (con sellos como Editorial Planeta, que incluía Espasa, Destino, Seix Barral y Temas de Hoy), se había estado abriendo un hueco en el sector de los medios de comunicación durante los últimos años con gran ahínco, logrando extenderse y estar presente de forma significativa en varios medios: socio de referencia de Audiovisual Española 2000 (empresa editora del diario La Razón), compartía con Onda Rambla (Luis del Olmo) una licencia de radio digital terrestre, filial Planeta 2010 dedicada a la producción de canales temáticos (proveedor de la fracasada plataforma de televisión digital terrestre Quiero TV), entre otras.

3. Tal fue el caso de la pauta única publicitaria, es decir, la emisión de los mismos contenidos publicitarios en todos los canales digitales del grupo al mismo tiempo así como su comercialización también conjunta. De esta forma, se gana en rentabilidad al vender un porcentaje ma-yor de audiencia (mediante la suma de todos los canales del Grupo) al precio de la cadena con mayor GRP. Los espacios publicitarios de los canales digitales (Neox, Nova y Nitro, en este caso) si fuesen vendidos por separado tendrían un precio de venta más bajo y se perderían bene-ficios.

4. Con el número 11843, la compañía controlada por el grupo Planeta se planteaba progresar en la diversificación de sus fuentes de ingresos, para reducir su dependencia de las fluctuaciones del mercado publicitario. Debemos hacer constar en este punto que el mercado de información telefónica está liberalizado en España desde el año 2002 y en él operaban en este momento hasta un total de 16 empresas, que sumaban una facturación cercana a los 70 millones de euros.

5. Sobre los usos de la telefonía móvil como ventana de consumo de contenidos televisivos Cfr. Vacas Aguilar, F. "Telefonía móvil: la cuarta ventana". Revista Zer, 23, 2007, pp. 199-217.

6. Desde Antena 3 Multimedia también se optó por apoyar las iniciativas sociales del Grupo, por lo que la plataforma Ponle Freno para la señalización de Puntos Negros y Tramos Peligrosos logró concentrar a través de la web las firmas solicitadas a los ciudadanos. 


\section{Referencias}

\section{Bibliografía}

- Antena 3 Televisión (2004). Informe anual 2003. San Sebastián de los Reyes: Antena 3.

- Antena 3 Televisión (2005). Informe anual 2004. San Sebastián de los Reyes: Antena 3.

- Antena 3 Televisión (2006). Informe anual 2005. San Sebastián de los Reyes: Antena 3.

- $\quad$ Antena 3 Televisión (2007). Informe anual 2006. San Sebastián de los Reyes: Antena 3.

- $\quad$ Antena 3 Televisión (2008). Informe anual 2007. San Sebastián de los Reyes: Antena 3.

- Antena 3 Televisión (2009). Informe anual 2008. San Sebastián de los Reyes: Antena 3.

- Antena 3 Televisión (2010). Informe anual 2009. San Sebastián de los Reyes: Antena 3.

- Coller, X. (2005). Estudio de casos. Madrid: CIS (Centro de Investigaciones Sociológicas).

- García Mirón, S. "Televisión y redes sociales: presencia de la estrategia 3.0 de Antena 3 en Facebook". En Ortega, F.; Cardeñosa, L. Nuevos Medios, Nueva Comunicación. Salamanca: II Congreso Internacional Comunicación 3.0, 2011, pp. 85-104.

- García Mirón, S. "Las emisoras tradicionales frente al desafío de Internet: la integración estratégica de Antena 3”. En León, B. (coordinador). La televisión ante el desafío de Internet. Salamanca: Comunicación Social Ediciones y Publicaciones, 2012, pp. 61-76.

- Legerén, Beatriz (2014). "Defiance. De la historia a la experiencia”, en Ubierna Gómez, F. \& Sierra Sánchez, J. Miscelánea sobre el entorno audiovisual en 2014. Madrid: Fragua, pp. 567-582.

- Martínez, M. M. (2006). "La investigación cualitativa (síntesis conceptual)”. Revista IIPSI. Volumen 9, $n^{\circ} 1$, pp.123-146.

- Pérez y Pérez, R. \& Castellanos Cerda, V. (2013). "Ya no se cuentan las historias como antes: transformación de las narrativas en la era digital”. ALAIC, Revista Latinoamericana de Ciencias de la Comunicación, vol. 10, no 19 (10), pp. 66-75.

- $\quad$ Reyes, P. y Hernandez, A. (2008). "El Estudio de Caso en el contexto de la Crisis de la Modernidad”. Cinta Moebio 32, pp. 70-89, www.moebio.uchile.cl/32/reyes.html
- Scolari, C. (2008). Hipermedicaciones. Elementos para una teoría de la comunicación digital interactiva. Barcelona: Gedisa.

- Vacas Aguilar, F. (2007). "Telefonía móvil: la cuarta ventana”. Revista Zer, 23, pp. 199-217.

\section{Hemerografía}

- “Antena 3 inicia el rodaje de "El inquilino", su serie marciana” (2004), ABC, 26 de mayo, p. 107.

- "Antena 3 inicia la nueva temporada de Aquí no hay quien viva" (2006), La Vanguardia, Comunicación, 6 de abril, p. 10 .

- "Antena 3 intenta recuperar el liderazgo del domingo con Mis adorables vecinos" (2004), $A B C, 7$ de abril, p. 87.

- "Antena 3 retira la serie "Divinos" al tercer episodio por su baja audiencia" (2006), ABC, 12 de julio, p. 112.

- "El estreno de "Mis adorables vecinos" se impone a "7 vidas" (2004), ABC, 13 de abril, p. 84.

- $\quad$ "Fernando Tejero salta a Antena 3 y ficha por El síndrome de Ulises" (2008), ABC, 26 de enero, p. 109.

- $\quad$ "La familia Mata y una más: Ana Ruiz ficha por la serie de Antena 3" (2008), ABC, 13 de noviembre, p. 108.

- "Tiempo de seriales. La ficción de producción propia copa la programación "El internado, el estreno nacional más visto desde 2005” (2007), ABC, 26 de mayo, p. 108 .

- “televisiva” (2005), La Vanguardia, Vivir, 7 de agosto, p. 7.

- “TVE, en caída libre por octavo mes" (2006), La Vanguardia, Sociedad, 2 de agosto, p. 30.

- "Una serie sobre un vecindario, apuesta de Antena 3 para la noche del domingo" (2003), ABC, 5 de septiembre, p. 83.

- "Vuelve la serie Los protegidos" (2012), ABC, 8 de marzo, p. 102.

\section{Referencias electrónicas}

- "A tortas con la vida: Antena 3 vuelve a apostar por el humor de José Luis Moreno", en ABC, 30/08/2005, en $\mathrm{su} \quad$ edición online http: / / www.abc.es/hemeroteca/historico-30-082005/abc/Comunicacion/a-tortas-con-la-vida-antena-3- 
vuelve-a-apostar-por-el-humor-de-jose-luismoreno_61517429735.html [20/12/2012].

- Amoroso, S.: "Entrevista con Luis Villanueva, presidente \& CEO, Venevision International: La clave es la sinergia"

http: / / www.cisneros.com/Entrevistas/Entrevista-conLuis-Villanueva-Venevision-International-La-clave-es-lasinergia [02/07/09].

- “Antena 3.0 la nueva televisión multimedia” en El Mundo, 08/05/2009, en su edición online http://www.elmundo.es/elmundo/2009/05/07/comun icacion/1241714588.html [16/04/2013].

- "Antena 3 estrena en enero la serie Doctor Mateo", en el portal televisivo Fórmula TV, 16/12/2008, http: / / www.formulatv.com/noticias/9750/antena-3estrena-en-enero-la-serie-doctor-mateo/ [30/03/2013].

- "Antena 3 finalizará la tercera temporada de $L a$ familia Mata con 10 episodios", en el portal televisivo Fórmula TV, 22/01/2009, http: / / www.formulatv.com/noticias/10108/antena-3finalizara-la-tercera-temporada-de-la-familia-mata-con-10episodios/ [29/03/2013].

- “Antena 3.0 la nueva televisión multimedia" en El Mundo, 08/05/2009, en su edición online http: / /www.elmundo.es/elmundo/2009/05/07/comun icacion/1241714588.html [16/04/2013].

- $\quad$ "Antena 3 emite esta noche el final de Los protegidos”, en la página online del diario Deia, 13/06/2012, http: / / www.deia.com/2012/06/13/ocio-y-

cultura/comunicacion/antena-3-emite-esta-noche-elfinal-de-39los-protegidos39 [30/03/2013].

- "Audiencias El internado", en el portal televisivo Fórmula TV, http://www.formulatv.com/series/elinternado/audiencias/ [01/04/2013].
- "De la gracia en los cataplines", en el diario digital 20minutos.es, $21 / 05 / 2007$ http:/ /www.20minutos.es/columna/236726/0/gracia/c ataplines/gracia/ [28/03/2013].

- "El internado se convierte en un videojuego para Nintendo DS", en la revista online de videojuegos Vandal.net, 18/03/2009, http: / / www.vandal.net/noticia/37877/el-internado-seconvierte-en-un-videojuego-para-nintendo-ds / [01/04/2013].

- "El último capítulo de Doctor Mateo este domingo", en La Vanguardia, 15/07/2011, en su edición online http: / /www.lavanguardia.com/television/20110715/54 186741414/el-ultimo-capitulo-de-doctor-mateo-estedomingo.html [30/03/2013].

- "Emilio Aragón se desmelena en la segunda temporada de Casi perfectos", en El País, 26/11/2004, en su edición online http: / / elpais.com/diario/2004/11/26/radiotv/1101423 601_850215.html [15/12/2012].

- "Manolo y Benito despiden temporada con su mínimo de audiencia, en el portal televisivo Fórmula $T V$, 13/03/2007,

http: / / www.formulatv.com/noticias/3967/audienciasmanolo-y-benito-despiden-temporada-con-su-minimo-deaudiencia/ [29/03/2013].

- Piña, R. "Telecinco demanda al portal de vídeos Youtube por piratear sus programas" en El Mundo, 19/06/2008, en su edición online http: / /www.elmundo.es/elmundo/2008/06/19/comun icacion/1213874329.html

[15/04/2013].

\section{Forma de Citación}

GARCÍA MIRÓN, Silvia: La aplicación de posibilidades transmedia e interactivas en las producciones seriadas de ficción de Antena 3: Análisis de la etapa Planeta (2003-2010). Revista Communication Papers, N ${ }^{\circ}$ 4, páginas 22 a 36. Departamento de Filología y Comunicación de la Universidad de Girona. Recuperado el__de de 2 de: http://www.communicationpapers.es 